\title{
Non-covalent chiral fibres in aqueous gels and their functionalization
}

\author{
JÜRGEN-HINRICH FUHRHOP*, SÖNKE SVENSON, CHRISTOPH BÖTTCHER, \\ OTTO TRÄGER, CORINNA DEMOULIN, PETER SCHNIEDER, \\ CHRISTIAN MESSERSCHMIDT, LAURENT RUHLMANN and JÖRG ZIMMERMANN \\ Institut für Organische Chemie, der Freien Universität Berlin, Takustraße 3, D-14195 Berlin, Germany
}

\begin{abstract}
The fully reversible synthesis of noncovalent assemblies which are held together by directed bonds is called synkinesis. Micellar fibres with a distinct stereochemistry and gels are formed by amphiphiles forming strong hydrogen bonds between chiral head groups. The 3D-crystallization of such molecular assemblies, without dominating repulsive hydration forces, is prevented by the curvature of the fibres. Curvature might perhaps be directly connected with chirality. Addition of enantiomers often leads to an irreversible destruction of gels. Recent developments relating to 'hydrophobic water' in Ångström-wide membrane gaps are also shortly discussed.
\end{abstract}

Keywords. Micellar fibres; porphyrin assemblies; aqueous gels.

\section{Introduction}

Hydrogels are usually made of chiral, polymeric fibres, e.g. $0.2 \%$ agar or $0.6 \%$ gelatine in up to $99.9 \%$ water. The solid fibres form a coherent net and are tightly connected at crossing points. Such hydrogels constitute large parts of biological organisms and are stable for many years under appropriate conditions, which is exemplified by the life-long transparency of the vitreous humour of the human eye.

Similar gels can also be made of low-molecular weight amphiphiles, provided they have chiral head groups which strongly interact with each other in aqueous media. Such amphiphiles do not form the usual spherical micelles or vesicles, but they appear as helical fibres or as long tubules with chiral surfaces. These fibres form slightly opaque hydrogels, which may live for years provided the formation of crystal seeds is prevented. Major singularities of these non-covalent fibres are: (i) the total reversibility of their formation, and (ii) the possibility to use reactive dyes as hydrophobic core of the fibre. The fully reversible synthesis of noncovalent assemblies which are held together by directed bonds is called synkinesis (Fuhrhop et al 1994a). One related fibre which is not held together by head group-head group binding interactions, namely the viscoelastic ephedrinium myristate fibre is also discussed and compared to other viscoelastic fibres and the Scheibe complexes. These fibres neither form coherent nets nor solid gels.

\section{Tartaric amides}

Tartaric acid contains two chiral carbon atoms bearing hydroxy groups and two terminal carboxylate groups.

*Author for correspondence
Upon amidation of one of the carboxyl groups with dodecylamine one obtains an amphiphile, which is well water-soluble in monomeric form at $\mathrm{pH}>8$. At the $\mathrm{pk}_{\mathrm{a}}$ of the acid, however, objects of light microscopic dimensions appear, which are not crystalline. They float in water for months and rapidly dissolve, when the $\mathrm{pH}$ is again raised above 8 . They precipitate as crystals below $\mathrm{pH}$ 3. If examined by electron microscopy, one observes thousands of circular micellar fibres which are interconnected to form cloth-like structures and which later closed to form wide tubules of statistical structure. The half-neutralized acids presumably form strong hydrogen bond chains between individual micellar strands. The whole structure constitutes an isolated gel body in bulk water (figure 1). Figure 1 reveals that the gel particle shows the most simple way of gel formation, namely parallel arrangement and manifold hydrogen bond cross linking of the single strands. Curvature of the fibres totally depends on chirality, although no helicity can be detected in TEMs.

\section{Open-chain carbohydrate fibres}

Open-chain hexose amides contain four chiral centres instead of the two in tartaric amides. In addition to the 1,4-Pitzer strain in the tartrate head group, there exists an even more important 1,5-Newman strain between hydroxyl groups, which leads to a variety of flexible conformations. Mannon- and galactonamides keep their linear conformations in fibres, because there is no 1,5 -Newman strain in the monomers. Their supramolecular assemblies therefore correspond to planar sheets. In case of mannonamide the sheets first roll up to form cigar-like structures. The curvature of these structures and the tendency to intertwine is low. Gels 
are therefore whitish and short-lived. Galactonamide sheets, on the other hand, appear as twisted ribbons, which yield perfect gels in the presence of SDS-micelles (figure 2).

Supramolecular structures of the bimolecular, micellar fibres are only found in aqueous media. Organogels of the same gluconamides only produce bulky fibrous assemblies with no defined diameter or helical pitch. Only after hydration of the head groups there is separation of the bilayer fibres from each other. In particular, the ${ }_{2} G^{-}$bend in glucon head groups leads to a strong hydration of the fibre surface and helix formation. In electron microscopy one observes quadruple micellar helices with a regular pitch of $22.4 \mathrm{~nm}$ and a magic angle against the axis of $55^{\circ}$. These fibres form slightly turbid gels at concentrations of $0 \cdot 1-2 \%(w / w)$. In pure water, rapid crystallization in form of head-to-tail layers occurs. $D$-gluconamide forms left-handed helices, $L$-gluconamide gives right-handed fibres. The racemic $D, L$-gluconamide only yields platelets which precipitate from hot micellar solutions upon cooling. The fibre and gel formation are thus again dependent on chirality. The gel also depends on strong connections between the fibres, which occurs in micellar knots at the crossing points. It is actually observed in time-dependent electron microscopy, that the first event in the rearrangement of spherical micelles in hot aqueous solution to fibres in

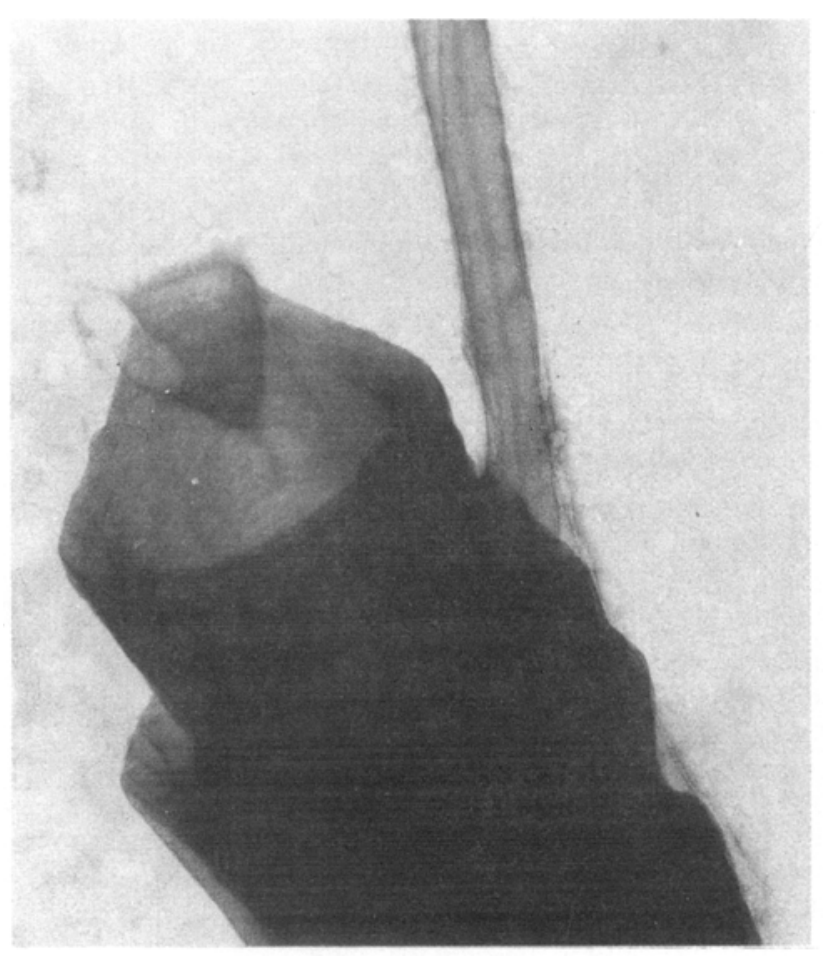

Figure 1. Transmission electron micrograph (TEM) of an isolated gel particle made of $N$-dodecyl-S,S-tartaric amide (Fuhrhop and Bottcher 1990). room-temperature gels is the clustering of the micelles (figure 3). From the large clusters the fibres then grow by cooperative formation of hydrogen bond chains only in a linear fashion. Further agglomerization only occurs in organic solvents. These gels live for years, if SDSmicelles are present in the bulk aqueous medium, which then dissolve crystal seeds made of monomers which are in equilibrium with the fibre.

The time course of the rearrangement of these fibres to crystals can be followed by light microscopy, provided one uses a limited amount of SDS. In this case, one first observes the formation of onion-type vesicles from
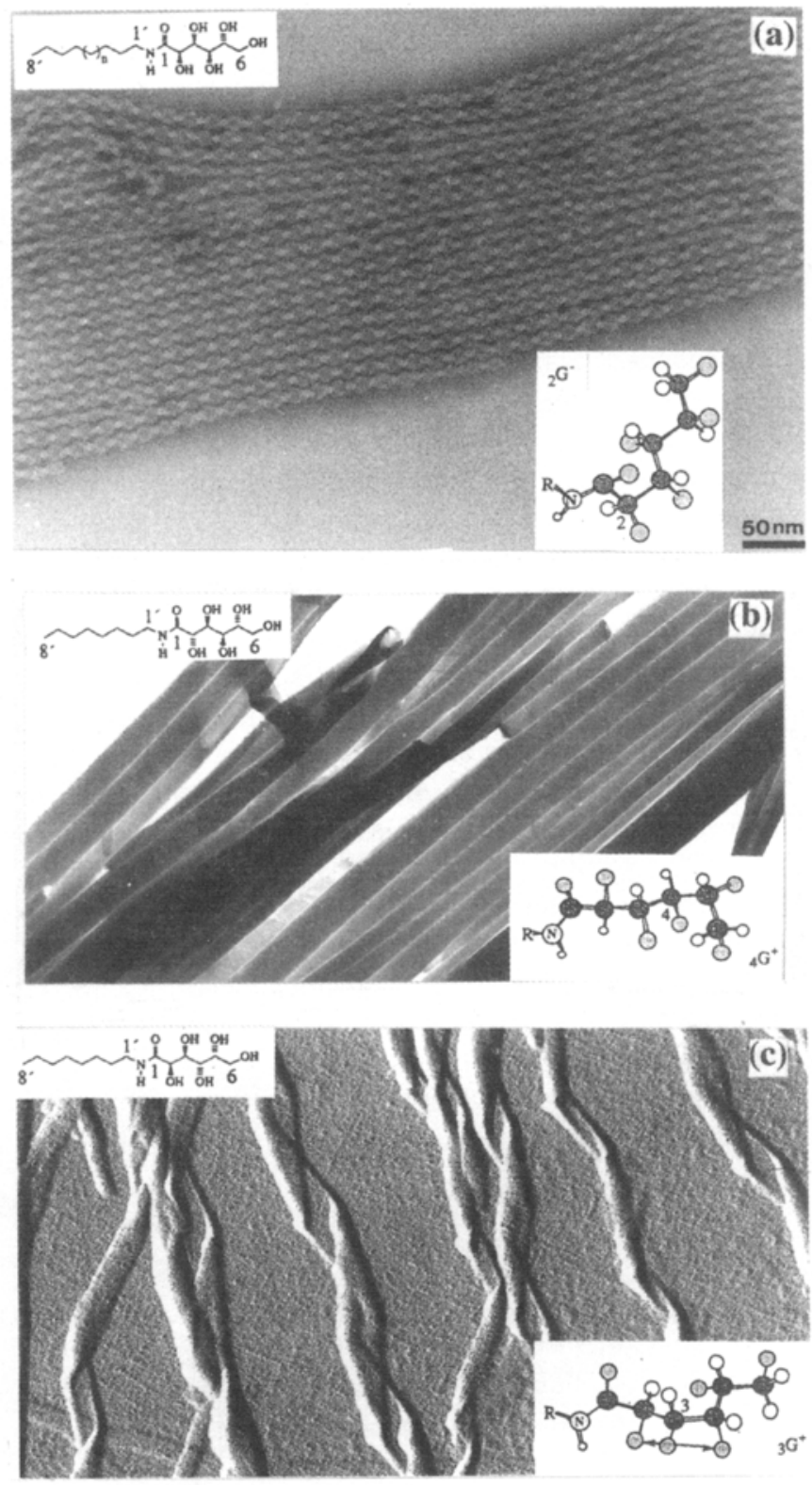

Figure 2. Typical conformation of (a) glucon-, (b) mannonand (c) galactonamides. $\mathrm{R}=$ octyl or dodecyl (Svenson et al 1994). 
the micellar fibres. These then swell to give myelin-figures after a period of about 3 months. After more than six months, lamellar assemblies without curvature have formed. Precipitation of 3D crystals begins (figure 4).

In attempts to functionalize the most stable gluconamide fibres as electron conducting wires, it was repeatedly tried to load them with redox active dyes. This was only successful in the case of gluconamide derivative of a carotenoid-polyene called bixin (Fuhrhop et al 1990b). This linear dye amphiphile gave yellow gels in a mixture with the quadrupole helices showing strong circular dichroism. It was not possible to integrate

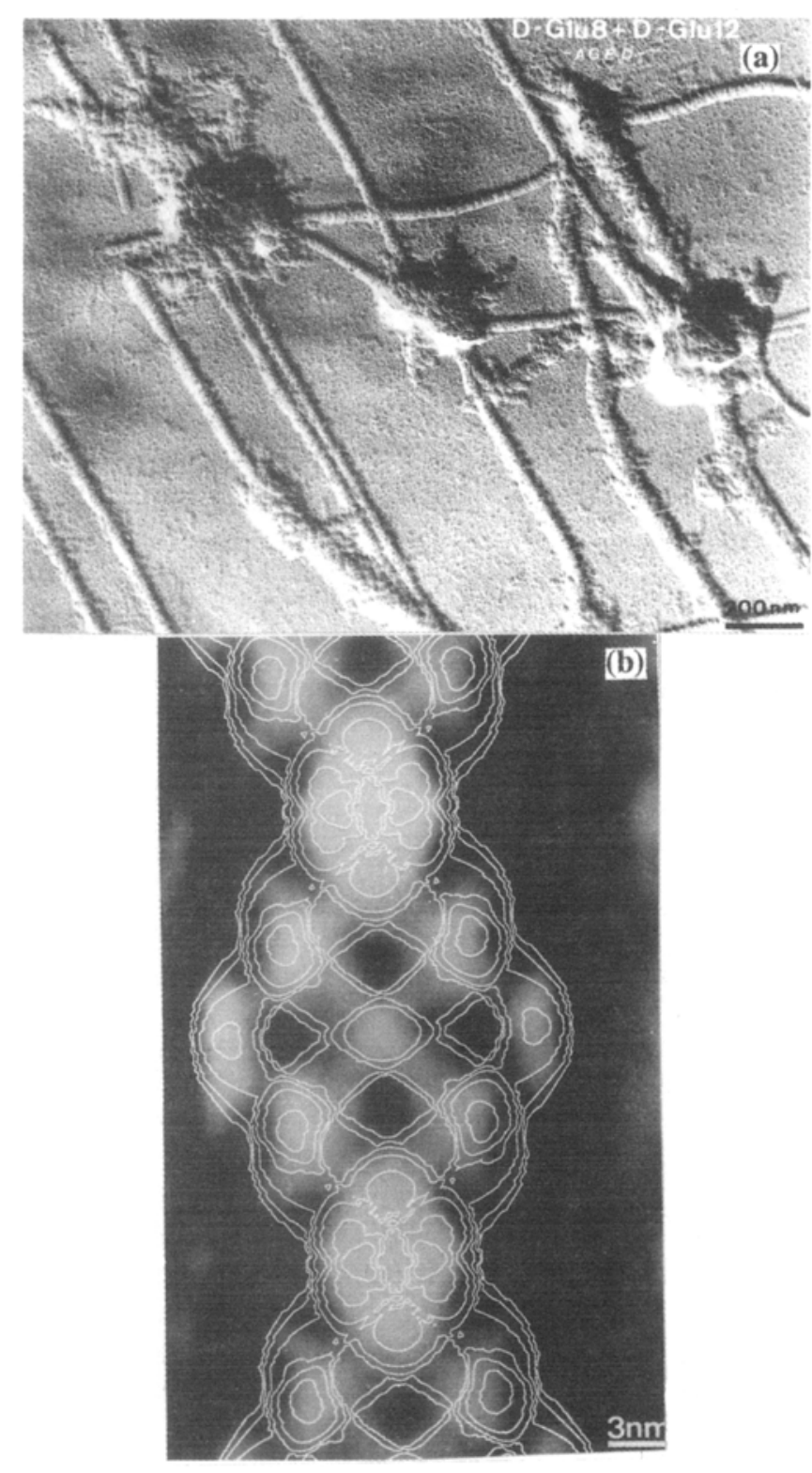

Figure 3. (a) Formation of octyl-D-gluconamide helices from large micellar clusters in hot aqueous solutions. (b) TEM of a typical quadruple helix after image analysis (Köning et al 1993). corresponding porphyrins into any of the tartaric amide or gluconamide assemblies. Amphiphilic or hydrophobic porphyrins dissolved beautifully in the heated micellar solutions, but upon cooling they were ejected from the fibres which formed. It was, however, found that the amphiphilic porphyrins form fibres and gels by themselves.

\section{Porphyrin gluconamide fibres}

Protoporphyrin IX, the all important mother compound of the hemes used in hemoglobins and cytochromes, has never been crystallized. This dye molecule with hydrophobic north, east and west edges and a hydrophilic south is an amphiphile which forms unstable fibres instead of crystals if precipitated from water. Stabilization of the fibres is again observed if the hydrophilic propionic acid groups on the south edge are derivatized with gluconamide. The porphyrin and its metal complexes, in particular the tin(IV) complex, then form fibres of bimolecular thickness and a deep-red hydrogel appears (figure 5a,b; Fuhrhop et al 1994). The Soret band is split in two bands with a separation of about $100 \mathrm{~nm}$ by exciton coupling and $\mathrm{CD}$-bands with molar ellipticities of $1.4 \times 10^{6} \mathrm{deg} \mathrm{M}^{-1} \mathrm{~m}^{-1}$ are found. The electric properties of these metalloporphyrin 'wires' are not yet known. Similar tin (IV) porphyrin gels are also obtained, if one uses chiral lactate counterions to the central tin(IV) porphyrin (Rosengarten et al 1998).

\section{Ephedrinium myristate viscoelastic fibres}

As a final example of a fibre which is dominated by chirality, we mention the ephedrinium myristate. The corresponding sodium salt also appears as fibre of light microscopic dimension, but it is just a rolled-up sheet of a myristate monohydrate. No dye is dissolved by this crystalline material. If, however, ephedrinium ions containing two chiral centres are used as a counterion, one obtains a fluid micellar fibre of $13 \mathrm{~nm}$ width. This fibre dissolves magnesium octaethylporphyrin, gives totally transparent and infinitely long-lived fibres. The solution is, however, not gel-like, it only shows viscoelasticity. No coherent net is formed by the fluid fibres. Again no fibres are formed, if both enantiomers of the ephedrinium cations are present in the solution (figure 6).

\section{Synkinesis and chirality effects}

The effects of chirality in biological gels have not been studied so far. It has obviously been too cumbersome to synthesize a collagen-like polymer from $D$-configured amino acids. It is clear however that all gels in the vitreous humour on the inner cell surface, in cartilage, in fruits etc are formed by helical biopolymers in 

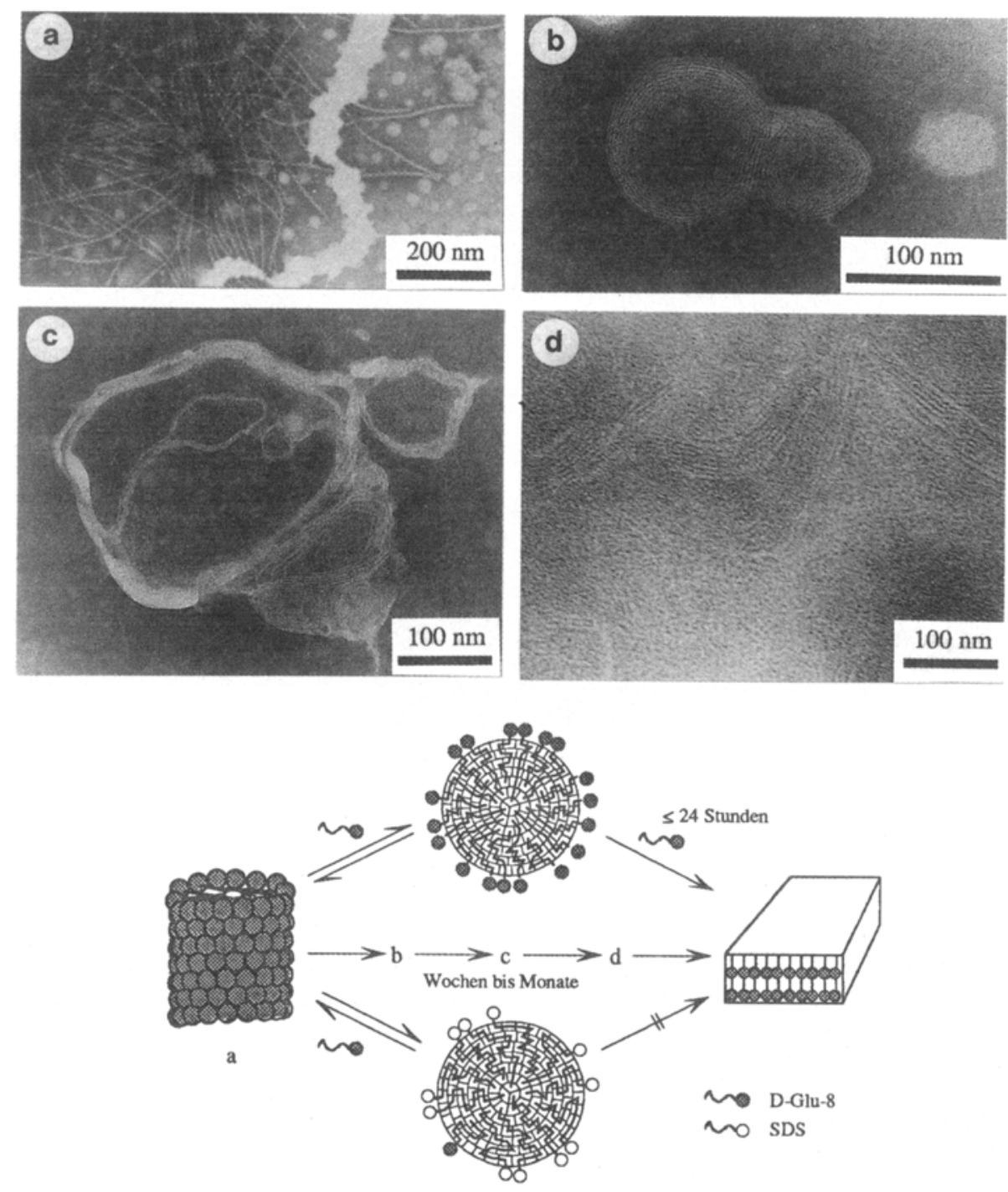

Figure 4. Transmission electron microscopic (TEM) pictures of $N$-octyl- $D$-gluconamide fibres in gels: (a) after a few hours, (b) after 6 weeks, (c) after 12 weeks, and (d) after 30 weeks. $2 \%$ of the gluconamide, $0 \cdot 2 \%$ SDS (w/w) stained on the grid with phosphotungstate (Fuhrhop and Böttcher 1990).

particular proteins and polysaccharides. In the case of synthetic $D$-, and $L$-poly(lysins) at $\mathrm{pH} 12$ it was shown that helices immediately precipitated as pleated sheets, when the aqueous solutions of $P$-and $M$-helices were mixed. One may expect that the enantiomeric helices and the hydrogels formed by them do not survive addition of enantiomers. This is however not applicable to nucleotides, where $D$-, and $L$-double helices add end-on, and no sheet structure is formed (Doi et al 1993). Furthermore, there is a large variety of nonchiral inorganic gels as well as of organic organogels. In general, the chirality effects only come into play if the fibres are ultrathin, mostly bimolecular, and the building blocks are partly water-soluble. Crystal sheet formation presumably does not take place by a direct intertwining of the mirror image helices, but by formation of racemic crystallites from dissociated monomers or small aggregates. Then it is found that the racemate crystals are often less soluble by two or three orders of magnitude, because face-to-face hydrogen bonding or similar dipolar interactions and symmetry are favoured. The fundamental example is tartaric acid which is more than a hundred times more soluble in water than racemic acid, because the latter forms a hydrogen bond cycle between the hydroxyl groups of the enantiomers (Parry 1951; Lehmann et al 1990).

The most important effect of chirality is that the asymmetry of each carbon atom leads to a regular displacement of the substituents of neighbouring carbon atoms. This is true in many covalent polymers as well 

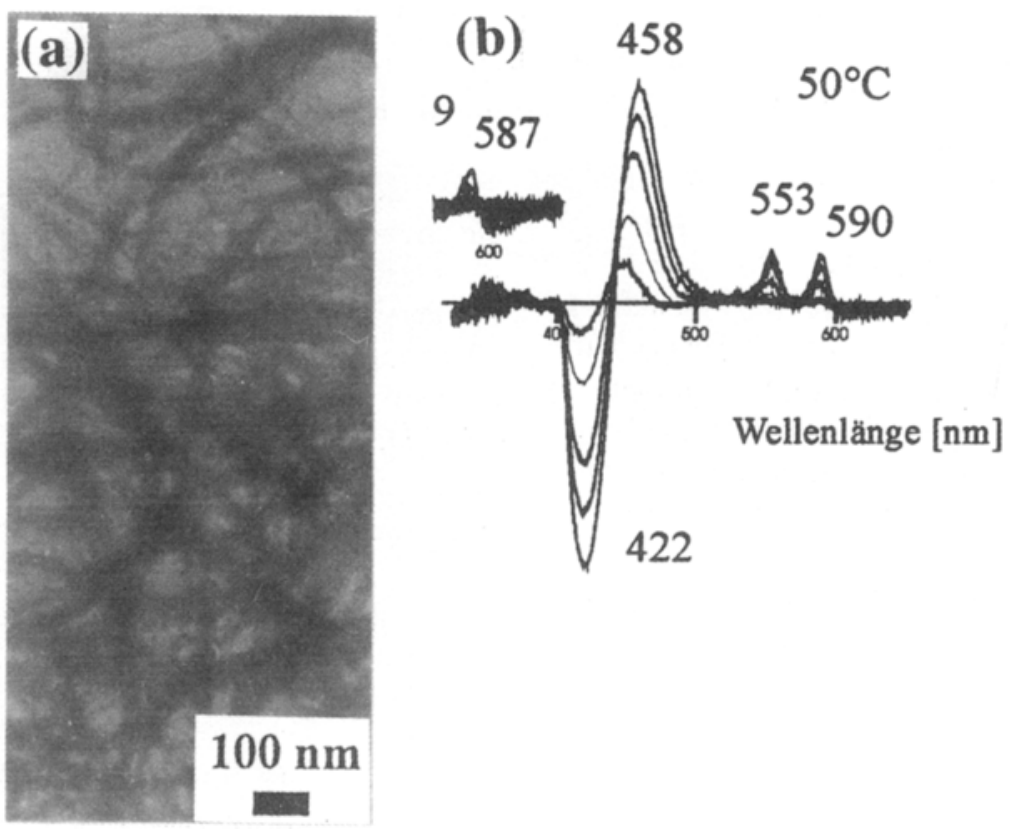

Figure 5. (a) TEM of a tin (IV) porphyrinate gluconamide fibre and (b) typical $\mathrm{CD}$-spectra of metalloporphyrin fibres.

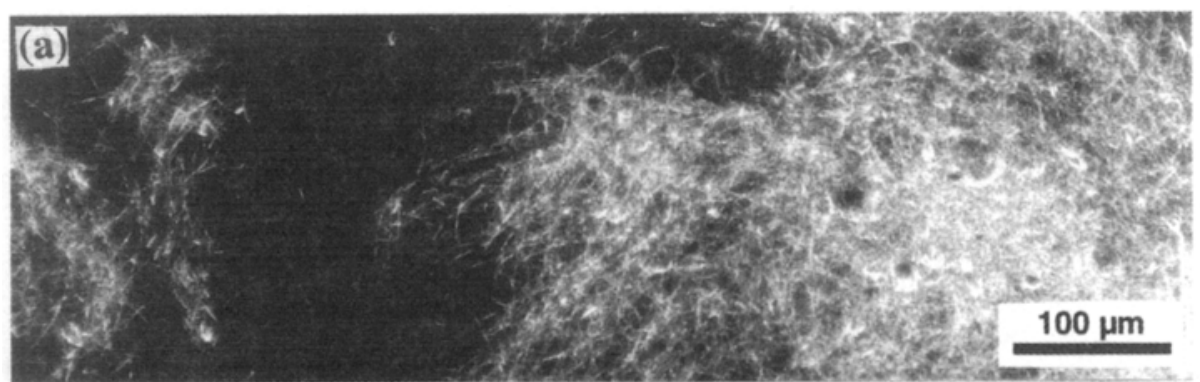

(b)

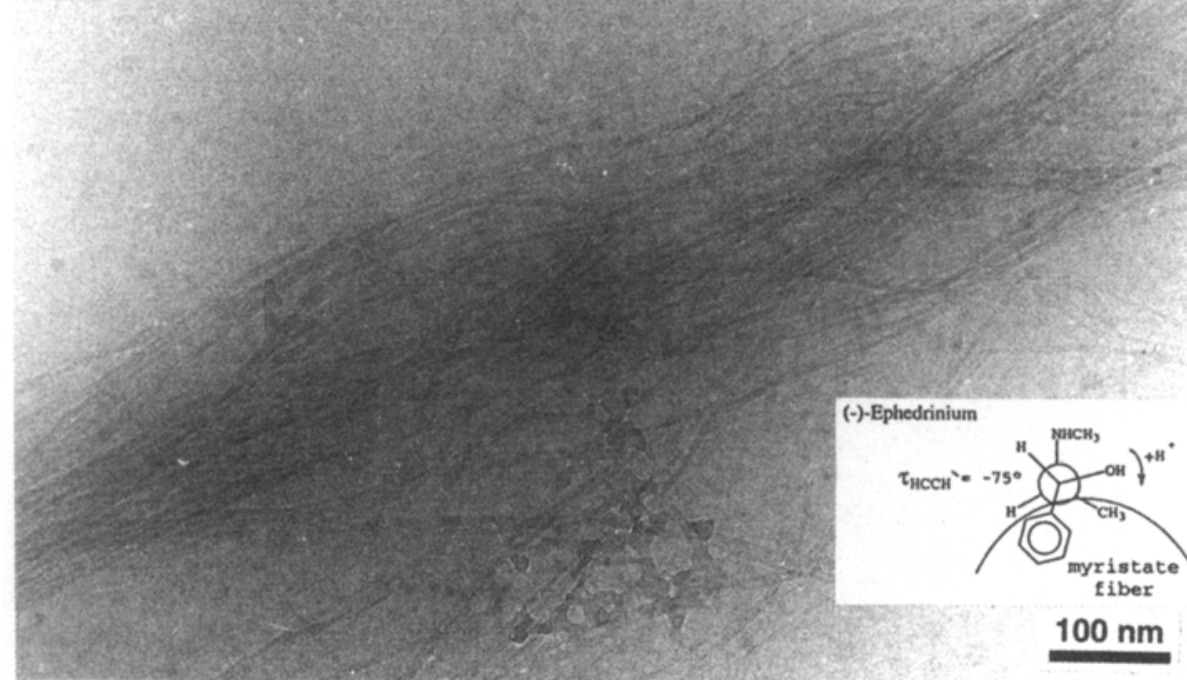

Figure 6. (a) Light micrograph and TEM of sodium myristate monohydrate fibres and (b) TEM of ephedrinium myristate fibres (Träger et al 1997). 
as in molecular assemblies, which are connected by direct non-covalent bonds. Such chiral effects should play no role in fluid micellar or vesicular assemblies where the head groups are disconnected in a nondiscriminatory manner by hydration forces.

Chirality alone, however, produces only helical displacements of bulky substituents, not curvature. A large variety of 3D-crystals contains helical substructures. For fibre formation in aqueous media it is mandatory that despite the strong binding interactions between the monomers there is enough space left for the entrapment of water molecules which are connected with the molecules in the bulk water volumes. This means strongly bent head groups, which have been analyzed for the first time in detail by solid state ${ }^{13} \mathrm{C}$-NMR spectroscopy in the glyconamide case (see figure 2). Similar bends will occur in amphiphiles with amino acid head groups, but not with nucleotides which have no flexible chain elements in them.

\section{7. Ångström gaps in membranes}

The phenomenon of gel formation in water depends on capillary effects. Water is immobilized on the surface of fibres, and if the fibres are immobilized by formation of coherent networks, the bound water is also immobilized. A related effect is observed in membrane gaps with the width of a few Angströms on gold surfaces. If such gaps are filled with water and rigid solutes, which fit into crystalline ice, the water behaves like an entrapped solid and does not exchange with bulk water within several days. These ice-bucket like membrane gaps are currently investigated as models for enzyme clefts. They provide unique possibilities to control the arrangement of molecular assemblies: molecules appear in the order of addition from the bulk solution. One starts, for example, with a polyanionic porphyrin which. is bound to a smooth gold surface. A rigid wall is then built around the porphyrin, and an oligo-cationic porphyrin or dimethyl viologen is added. Next, an anionic porphyrin or quinone can be filled into the membrane gap. The success of this procedure heavily depends on the gel like behaviour of the water volume. Its immobility prevents equilibration with the bulk water (Fuhrhop et al 1997; Ruhlmann 1998).

\section{Conclusions}

The functional porphyrin assemblies should be useful as electron-wires after appropriate doping and ordering. The gel clusters in fluid bulk water may be used as carrier for heavy metal or other biologically-active ions. Since all of the assemblies discussed in this article are simple derivatives of common natural compounds, they will all be biodegradable.

\section{References}

Doi $\mathrm{M}$, Inoue $\mathrm{M}$, Tomoo $\mathrm{K}$, Ishida $\mathrm{T}$, Ueda $\mathrm{Y}$, Akagi $\mathrm{M}$ and Urata H 1993 J. Am. Chem. Soc. 11510432

Fuhrhop J-H and Böttcher C $1990 \mathrm{~J}$. Am. Chem. Soc. 112 1768

Fuhrhop J-H and Köning J 1994 Molecular assemblies and membranes, in monographs in supramolecular chemistry (London: Royal Soc. Chem.) i-xiii, pp 1-227

Fuhrhop J-H, Demoulin C, Rosenberg J and Böttcher C 1990a J. Am. Chem. Soc. 1122827

Fuhrhop J-H, Krull M, Schulz A and Möbiuis D 1990b Langmuir 6497

Fuhrhop J-H, Bindig U and Siggel U 1994 Chem. Commun. 1583

Fuhrhop J-H, Bedurke T, Gnade M, Schneider J and Doblhofer K 1997 Langmuir 13455

Israclachvili J 1992 Intermolecular and surface forces (New York: Academic Press) p. 278

Köning J, Böttcher C, Winkler $\mathrm{H}$, Zeitler $\mathrm{E}$, Talmon $\mathrm{Y}$ and Fuhrhop J-H $1993 \mathrm{~J}$. Am. Chem. Soc. 115693

Lehmann C W, Buschmann J, Luger P, Demoulin C, Fuhrhop J-H and Eichbom K 1990 Acta Crystallogr. B46 646

Parry G S 1951 Acta Crystallogr. 4131

Rosengarten B, Böttcher C, Schulz A, Fuhrhop J-H and Siggel U 1998 J. Porphyrins Phthalocyanines 2273

Ruhlmann L, Zimmermann J, Roeder B, Fudickar W, Siggel U and Fuhrhop J-H 1999 (submitted)

Svenson S, Kirste B and Fuhrhop J-H 1994 J. Am. Chem. Soc. 11611969

Träger O, Sowade S, Böttcher C and Fuhrhop J-H $1997 \mathrm{~J}$. Am. Chem. Soc. 1199120 